\title{
The assessment of platelet function using multiple electrode aggregometry in practical procedures in anaesthesia
}

\author{
Jan Pluta ${ }^{1}$, Barbara Nicińska ${ }^{1}$, Michał Ciurzyński ${ }^{2}$, Janusz Trzebicki ${ }^{1}$ \\ 'I Department of Anaesthesiology and Intensive Care, Medical University of Warsaw, Poland \\ ${ }^{2}$ Department of Internal Medicine and Cardiology, Centre for Diagnosis and Treatment of Venous \\ Thromboembolic Disease, Medical University of Warsaw, Poland
}

\begin{abstract}
Background: Platelets are responsible for primary haemostasis. Patients with suspected platelet dysfunction require prompt clinical assessment when qualifying for emergency surgical procedures. The purpose of this article is to present our experience in platelet function assessment using whole-blood multiple electrode aggregometry (MEA) in various clinical conditions.

Case reports: Retrospective analysis of three patients with thrombocytopathy associated with normal platelet counts was performed using standard laboratory tests complemented by MEA. In two cases, platelet dysfunction was due to antiplatelet drugs, while in one other case it was caused by chronic kidney disease.

Conclusions: Anaesthesiologists strive to make the perioperative period as safe as possible. Platelet function assessment should be considered in every patient in whom haemostatic disturbances are suspected. MEA provides support for clinical decision-making, especially in patients who undergo haemodialysis or require antiplatelet therapy, and are in need of emergency surgery.
\end{abstract}

Anaesthesiology Intensive Therapy 2018, vol. 50, no 3, 210-214

Key words: haemostasis; thrombocytes, function, monitoring; thrombocyte activation, aggregation; multiple electrode aggregometry; thromboelastometry; point-of-care; POC; POCT

Platelets (PLT) are a significant element of the currently accepted cell-based model of haemostasis. Together with the vascular endothelium, they are involved in the formation of a platelet plug in response to discontinuity of a blood vessel [1]. A normal PLT count does not ensure effective primary haemostasis; their function is essential. However, the qualitative assessment of platelets is a major problem in everyday anaesthetic practice.

The aim of the study was to describe the use of multiple electrode aggregometry (MEA), a point-of-care method enabling the assessment of platelet function, in various clinical conditions. The detailed description of the method was presented in a previous paper by Pluta et al. [2].

The study design, i.e. a retrospective analysis of three cases, was approved by the Bioethics Committee of the Medical University of Warsaw (AKBE/195/16).

\section{CASE REPORT A}

An 88-year-old male patient, subjected earlier to a right lung lobectomy, with a history of inferior myocardial infarction and aorto-coronary bypass grafting with an implanted stimulating system due to the tachycardia-bradycardia (tachy-brady) syndrome, persistent atrial fibrillation with incomplete Morgagni-Adams-Stokes syndrome, and heart failure with an indirect left ventricular ejection fraction of $45 \%$, was admitted to the Department of Orthopaedics due to a transtrochanteric femoral fracture. The patient was scheduled for surgical management of the fracture. On hospitalisation day 2, while awaiting surgery, the patient reported chest pain with dyspnoea. A new non-ST-elevation myocardial infarction (NSTEMI) was diagnosed and the patient was transferred to the Department of Cardiology where he received typical pharmacological treatment, in- 
A

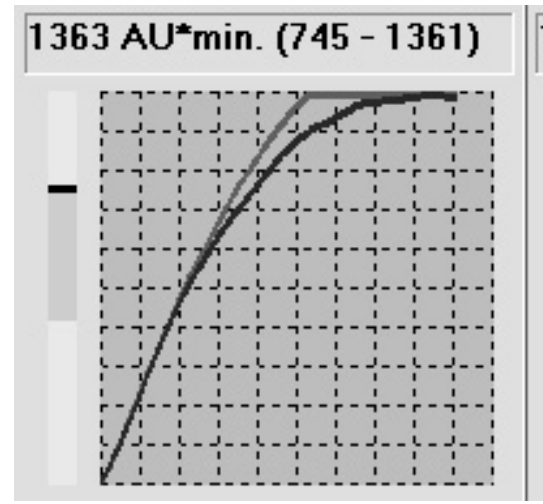

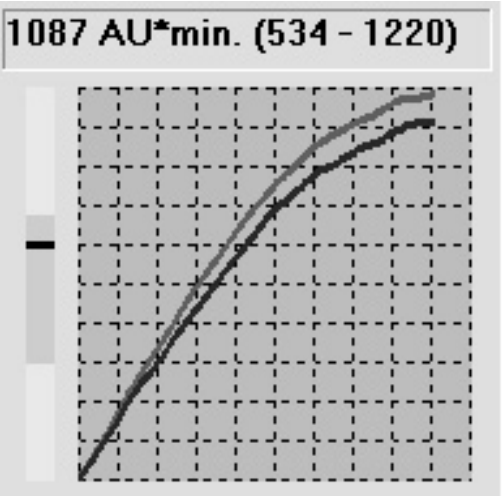

Figure 1. Graphical and numerical results of ASPItest (A) and ADPtest (B) performed before subarachnoid anaesthesia and surgery

cluding dual antiplatelet therapy with acetylsalicylic acid (ASA) and clopidogrel. Coronarography demonstrated critical stenosis of the diagonal branch 1 (DB1), which was managed with balloon angioplasty. The procedure was uneventful and the patient was transferred to the Cardiac Intensive Care Unit. Prior to surgical management of the fracture, cardiologists assessed the risk of perioperative cardiovascular complications as high, yet did not find absolute contraindications for surgery. Clopidogrel was discontinued and the patient was sent back to the Department of Orthopaedics for the surgical procedure to be carried out 7 days after the discontinuation of clopidogrel: ASA therapy and low-molecular-weight heparin in a prophylactic dose were to be continued. Due to the fear of intraoperative haemorrhagic complications, the orthopaedic team decided to discontinue ASA and continue low-molecular-weight heparin. Five days after the discontinuation of clopidogrel and three days after the discontinuation of ASA, considering the high risk of losing function in the broken limb associated with the further postponement of surgery (progressive perfusion disorders accompanied by severe pain), the orthopaedic team decided to perform an emergency procedure. A preoperative anaesthetic consultation was requested during which surgery under general anaesthesia was considered. However, due to the presence of numerous medical issues (post-myocardial infarction heart failure - NYHA III/IV, partially controlled bronchial asthma, the patient's condition after a right lung lobectomy), unilateral subarachnoid anaesthesia was decided upon. Since the time interval between the discontinuation of clopidogrel ( 5 days) and ASA ( 3 days) and the central block was shorter than that recommended, the standard laboratory tests (PLT 205 G $\mathrm{L}^{-1}$, coagulogram within the reference values) were supplemented with PLT function assessment using MEA. The following tests were performed: ASPItest (assessing the cycloxygenase-dependent pathway of PLT activation, susceptible to ASA and nonsteroidal anti-inflammatory drugs); and ADPtest (evaluating adenosine diphosphatedependent PLT activation, susceptible to clopidogrel, prasugrel and other ADP-P2Y12 receptor antagonists).

The MEA results demonstrated no anti-aggregative effects of either ASA or clopidogrel (Fig. 1).

\section{CASE REPORT B}

A 43-year-old female patient with end-stage renal failure due to glomerulonephritis, chronically dialysed, was qualified for a deceased-donor kidney transplant. The peripheral blood testing demonstrated anaemia $\left(109 \mathrm{~g} \mathrm{~L}^{-1}\right)$ and a normal PLT count $\left(196 \mathrm{G} \mathrm{L}^{-1}\right)$. The standard laboratory tests did not show any clotting abnormalities. The patient's history revealed a kidney biopsy complicated by massive bleeding requiring transfusion of blood preparations, which could have suggested congenital or acquired haemostasis disorders. The patient denied the occurrence any spontaneous bleedings or taking drugs affecting the function of the clotting system. Considering the incomplete information about the cause of bleeding and normal laboratory results, during the pre-operative anaesthetic consultation, the decision was made to perform thromboelastometry (ROTEM) (in order to assess the haemostatic potential in whole blood - the result was within the reference values) and MEA in order to assess the baseline function of PLT. The TRAPtest was applied, evaluating the TRAP-6-activated pathway of PLT aggregation. The above test is considered the most reliable for monitoring the overall aggregative potential of PLT in antiplatelet-naive patients [3]. The test result was 695 $\mathrm{AU}^{*} \mathrm{~min}$, i.e. lower than the reference range (Fig. 2).

\section{CASE REPORT C}

A 59-year-old female patient with numerous medical conditions, namely antiphospholipid syndrome, Sjögren`s syndrome, 


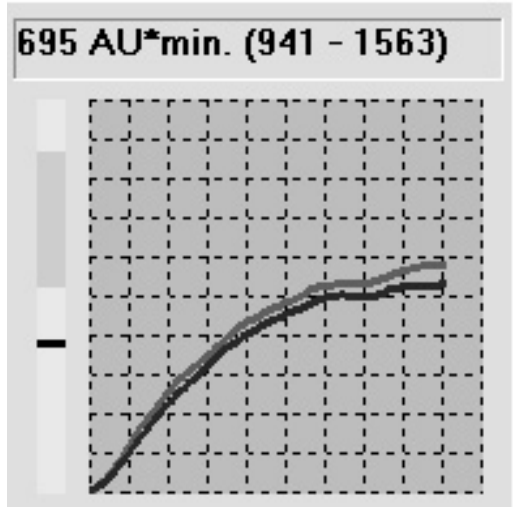

Figure 2. Graphical and numerical results of TRAPtest performed prior to kidney transplant

peripheral neuropathy, secondary hyperparathyroidism, and haemodialised since 2013 due to end-stage renal failure, presented to the Department of General and Transplant Surgery for qualification for a kidney transplant (living related donation).

The patient's history related with haemostasis disorders revealed ischaemic cerebral stroke (1997), myocardial infarction and angioplasty of the anterior interventricular branch (2009), right internal carotid endarterectomy, another myocardial infarction and the implantation of two drug-eluting stents (DES) (2013). Due to progressing ischaemic nephropathy, angioplasty of the right renal artery was performed.

According to the available medical records, after the 2009 episode of myocardial infarction the patient received dual antiplatelet therapy (ASA $75 \mathrm{mg}$ and clopidogrel 75 $\mathrm{mg}$ ). Despite the therapy applied, the patient developed an internal carotid artery occlusion; therefore, clopidogrel was replaced with prasugrel. The therapy did not prevent another myocardial infarction in 2013. Due to progressive renal failure and necessary haemodialyses, the consulting nephrologist recommended returning to treatment with clopidogrel combined with ASA in the doses administered earlier.
Considering the patient's positive history and a high risk of perioperative thromboembolic complications, an anaesthetic consultation was requested during which global assessment of the haemostasis system using thromboelastometry and PLT function assessment by MEA were decided upon. The ROTEM results were within the reference values. The MEA tests (ASPItest for ASA and ADPtest for clopidogrel) demonstrated an aggregation of 26 and $573 \mathrm{AU}^{*}$ min., respectively (Fig. 3).

The results show a lack of full efficacy of clopidogrel anti-aggregative effects with simultaneous complete block of the ASA-dependent pathway $[4,5]$.

\section{DISCUSSION}

The results obtained using the method described above demonstrated an actual aggregative potential of platelets. The use of only standard laboratory tests would not have been sufficient to comprehensively assess the haemostatic capacities of thrombocytes.

The first issue discussed was the use of antiplatelet drugs in patients scheduled for elective central blocks. A relevant problem in everyday anaesthetic practice is the lack of explicit recommendations regarding the time intervals between the final dose of antiplatelet drugs and subarachnoid or epidural anaesthesia. The suggested protocols are inconsistent [6]. In the group of patients receiving clopidogrel, a 7-day interval [7] or 5-day interval [8, 9] is most commonly recommended. Benzon et al. [10] suggest that in cases with a clopidogrel discontinuation and central block interval shorter than 7 days, platelet function should be assessed.

According to the 2012 guidelines of the Regional Anaesthesia Section of the Polish Society of Anaesthesiology and Intensive Therapy and the Polish Society of Regional Anaesthesia, clopidogrel should be discontinued 7 days before elective subarachnoid or epidural anaesthesia. The discontinuation of ASA, however is not required unless another antiplatelet or anticoagulant drug is additionally administered.
A

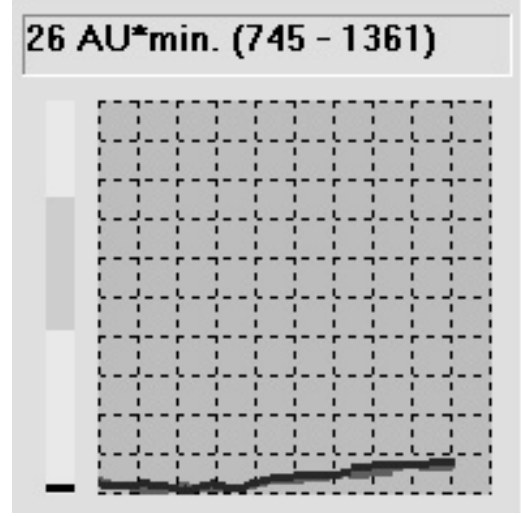

B

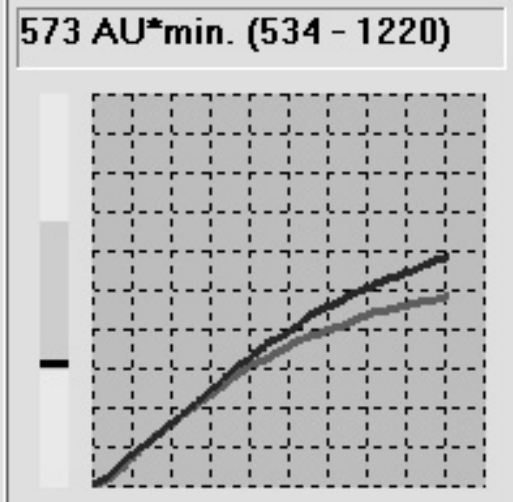

Figure 3. Graphical and numerical results of the ASPItest (A) and ADPtest (B) before kidney transplant from a relative in a patient receiving chronic dual antiplatelet therapy 
In our case A, in which an emergency procedure was needed and the risk of perioperative complications associated with general anaesthesia was high, it was decided to perform spinal anaesthesia following a qualitative assessment of PLT function using MEA. The literature reports describe the use of PLT function assessment in patients on antiplatelet drugs qualified for emergency surgical procedures [11]. Despite a shorter interval between clopidogrel discontinuation ( 5 days instead of the recommended 7 days) and between the final ASA dose (3 days) and subarachnoid anaesthesia, the results, i.e. ASPItest = 1363 (norm: 745-1361 $\mathrm{AU}^{*} \mathrm{~min}$ ) and ADPtest $=1087$ (norm: 534-1220 AU*min), were within the upper reference range, indicating normal PLT reactivity in both platelet haemostasis pathways. During the following post-procedure days, the patient was monitored neurologically for possible spinal haematoma. The perioperative period was uneventful.

PLT function assessment before the central block helped one to decide on the optimal form of anaesthesia to be administered in a given clinical condition.

The second of our cases is an example of the use of MEA in an antiplatelet-naive patient. Patients chronically dialysed are at risk of perioperative haemorrhagic complications due to acquired haemostasis disorders $[12,13]$. The implicated causes of thrombocytopathy include the pathological activation of PLT after their contact with the artificial surface of dialyser membranes, changes in glycoprotein expression on their surface and negative effects of uraemia [14].

MEA performed before the surgery enables one to determine the baseline PLT function and to monitor the changes in individual pathways of their activation and aggregation after therapeutic interventions, such as transfusions of PLT concentrates, use of tranexamic acid or desmopressin $[15,16]$.

In case $B$, the overall PLT function was impaired. In the case of intraoperative haemorrhage, thrombocytopathy resulting from chronic kidney failure and dialysis therapy should be an indication for considering the transfusion of PLT despite their proper count [17].

Patients on chronic antiplatelet therapy constitute a separate group. The available protocols of management enable its individualisation based on the MEA results, which was illustrated in our case $C$. The first stage involves the assessment of PLT activation after the initiation of therapy. An ADPtest result for clopidogrel below $500 \mathrm{AU}^{*}$ min proves the efficacy of the therapy $[5,18,19]$. If no such an effect is observed, and the lack of therapeutic adherence is excluded, the clinician should consider modification of the therapy. One of the options suggested is to increase the 24-hour dose of clopidogrel (from $75 \mathrm{mg}$ to $150 \mathrm{mg} 1 \times$ a day or 75 $\mathrm{mg} 2 \times$ a day). The efficacy of this modified therapy should be re-assessed after 1-2 weeks [4].
Possible modifications of ASA and clopidogrel therapy seem justified in our patient who developed further thromboembolic episodes, despite the use of standard dual antiplatelet therapy. The simultaneous use of aggregometry and thromboelastometry enables comprehensive evaluation of global haemostasis, including the PLT function [20]. Taking into account the result obtained and a history indicating a high risk of thromboembolic complications, the anaesthetic and surgical teams decided to postpone qualifying the patient for a kidney transplant until the information about the possibilities and efficacy of antiplatelet therapy modifications becomes available.

Despite the lack of explicit guidelines regarding routine PLT function assessment in patients with recurrent thromboembolic incidents, the use of MEA can help one to optimise antiplatelet therapy [21], in particular, to reduce the risk of perioperative thromboembolic complications.

\section{CONCLUSIONS}

The practical use of multiple electrode aggregometry enables individual assessment of the haemostatic potential. MEA provides quick and repeatable information about platelet aggregation, which determines the formation of a platelet plug, contributing to more effective evaluation of the risk associated with the invasive procedures performed.

In each of our cases, the decision about the anaesthetic management option was based on MEA, which is not possible when standard laboratory testing of the clotting system is performed.

The diversity of the cases presented reveals the clinical value of PLT function assessment. The unquestionable advantages of this method include its possible use before and after the initiation of antiplatelet therapy and repeatable assessment of the efficacy of the haemostatic therapy applied. Although MEA is still not a widely popularised method for PLT function monitoring, its use in certain groups of patients is recommended by European anaesthetic circles $[22,23]$.

\section{ACKNOWLEDGEMENTS}

1. Source of funding: none

2. Conflict of interest: none.

\section{References:}

1. Sokołowska B. Repetytorium z fizjologii hemostazy. Acta Haematol Pol. 2010; 2: 245-252.

2. Pluta J, Nicińska B, Trzebicki J. Multiple electrode aggregometry as a method for platelet function assessment according to the European guidelines. Anaesthesiol Intensive Ther. 2018 [Epub ahead of print], doi: 10.5603/AIT.a2018.0024, indexed in Pubmed: 30001456.

3. Ranucci M, Baryshnikova E, Soro G, et al. Surgical and Clinical Outcome Research (SCORE) Group. Multiple electrode whole-blood aggregometry and bleeding in cardiac surgery patients receiving thienopyridines. Ann Thorac Surg. 2011; 91(1): 123-129, doi: 10.1016/j. athoracsur.2010.09.022, indexed in Pubmed: 21172499.

4. Calatzis A, Loreth R, Spannagl M. Multiplate platelet function analysis - application and interpretation. V2.0/07.2007. Dynabyte Medical. 2007 Monachium. 
5. Sibbing D, Braun S, Morath T, et al. Platelet reactivity after clopidogrel treatment assessed with point-of-care analysis and early drug-eluting stent thrombosis. J Am Coll Cardiol. 2009; 53(10): 849-856, doi: 10.1016/j.jacc.2008.11.030, indexed in Pubmed: 19264241.

6. Vela Vásquez RS, Peláez Romero R. Aspirin and spinal haematoma after neuraxial anaesthesia: Myth or reality? Br J Anaesth. 2015; 115(5): 688-698, doi: 10.1093/bja/aev348, indexed in Pubmed: 26475800.

7. Horlocker TT, Wedel DJ, Rowlingson JC, et al. American College of Chest Physicians. Executive summary: regional anesthesia in the patient receiving antithrombotic or thrombolytic therapy: American Society of Regional Anesthesia and Pain Medicine Evidence-Based Guidelines (Third Edition). Reg Anesth Pain Med. 2010; 35(1): 102-105, doi: 10.1097/AAP.0b013e3181c15dd0, indexed in Pubmed: 20048659.

8. Breivik H, Bang U, Jalonen J, et al. Nordic guidelines for neuraxial blocks in disturbed haemostasis from the Scandinavian Society of Anaesthesiology and Intensive Care Medicine. Acta Anaesthesiol Scand. 2010; 54(1): 16-41, doi: 10.1111/j.1399-6576.2009.02089.x, indexed in Pubmed: 19839941

9. Benzon HT, McCarthy RJ, Benzon HA, et al. Determination of residual antiplatelet activity of clopidogrel before neuraxial injections. $\mathrm{Br} J$ Anaesth. 2011 ; 107(6): 966-971, doi: 10.1093/bja/aer298, indexed in Pubmed: 21968250

10. Benzon H. Regional anesthesia in the anticoagulated patient. NYSORA 2013. https://www.nysora.com/regional-anesthesia-in-anticoagulated-patient.

11. Herbstreit F, Peters J. Spinal anaesthesia despite combined clopidogrel and aspirin therapy in a patient awaiting lung transplantation: effects of platelet transfusion on clotting tests. Anaesthesia. 2005; 60(1): 85-87, doi: 10.1111/j.1365-2044.2004.04029.x, indexed in Pubmed: 15601279.

12. van Bladel $E R$, de Jager $R L$, Walter $D$, et al. Platelets of patients with chronic kidney disease demonstrate deficient platelet reactivity in vitro. BMC Nephrol. 2012; 13: 127, doi: 10.1186/1471-2369-13-127, indexed in Pubmed: 23020133.

13. Pluta J, Nicińska B, Grzeszczyk M, et al. Assessment of the hemostatic parameters and platelet function on thromboelastometry and impedance aggregometry in hemodialysis patients qualified for kidney transplantation: Preliminary Report. Transplant Proc. 2016; 48(5): 1431-1434, doi: 10.1016/j.transproceed.2016.02.057, indexed in Pubmed: 27496422.

14. Mądro E, Małyszko J. Coagulation disturbances in kidney diseases. Hematologia. 2011; 4: 332-338.

15. Weber CF, Dietrich W, Spannagl M, et al. A point-of-care assessment of the effects of desmopressin on impaired platelet function using multiple electrode whole-blood aggregometry in patients after cardiac surgery. Anesth Analg. 2010; 110(3): 702-707, doi: 10.1213/ ANE.0b013e3181c92a5c, indexed in Pubmed: 20042444.

16. Weber CF, Görlinger K, Byhahn C, et al. Tranexamic acid partially improves platelet function in patients treated with dual antiplatelet therapy. Eur J Anaesthesiol. 2011; 28(1): 57-62, doi: 10.1097/EJA .0b013e32834050ab, indexed in Pubmed: 20962655

17. Mishra PK, Thekkudan J, Sahajanandan R, et al. The role of point-of-care assessment of platelet function in predicting postoperative bleeding and transfusion requirements after coronary artery bypass grafting. Ann Card Anaesth. 2015; 18(1): 45-51, doi: 10.4103/0971-9784.148321, indexed in Pubmed: 25566711.

18. Jámbor $C$, von Pape KW, Spannagl M, et al. Multiple electrode whole blood aggregometry, PFA-100, and in vivo bleeding time for the point-of-care assessment of aspirin-induced platelet dysfunction in the preoperative setting. Anesth Analg. 2011; 113(1): 31-39, doi: 10.1213/ ANE.0b013e31821acddc, indexed in Pubmed: 21519054.

19. Roche. Multiplate analyzer: Cut-off-values ADPtest and ASPItest. 2013. http://www.cobas.com/content/dam/cobas_com/pdf/product/ Multiplate\%20tests/ADPtest-ASPItest-Pocket\%20guide\%20-Cut-off-values\%20.pdf.

20. Health Policy Advisory Committe on Technology. Rotational thromboelastometry (ROTEM ${ }^{\oplus}$ ) - targeted therapy for coagulation management in patients with massive bleeding. 2012. https://www.health.qld.gov. au/ data/assets/pdf file/0023/427145/wp024.pdf.

21. Tantry US, Bonello L, Aradi D, et al. Working Group on On-Treatment Platelet Reactivity. Consensus and update on the definition of on-treatment platelet reactivity to adenosine diphosphate associated with ischemia and bleeding. J Am Coll Cardiol. 2013; 62(24): 2261-2273, doi: 10.1016/j.jacc.2013.07.101, indexed in Pubmed: 24076493.

22. Kozek-Langenecker SA, Ahmed AB, Afshari A, et al. Management of severe perioperative bleeding: guidelines from the European Society of Anaesthesiology: First update 2016. Eur J Anaesthesiol. 2017; 34(6): 332-395, doi: 10.1097/EJA.0000000000000630, indexed in Pubmed: 28459785.

23. Rossaint R, Bouillon B, Cerny V, et al. The European guideline on management of major bleeding and coagulopathy following trauma: fourth edition. Crit Care. 2016; 20: 100, doi: 10.1186/s13054-016-1265-x, indexed in Pubmed: 27072503.

\section{Corresponding author:}

Jan Pluta

I Department of Anaesthesiology

and Intensive Care, Medical University of Warsaw

Lindleya 4, 02-005 Warszawa, Poland

e-mail:jan.pluta@lekarz.eu

Received: 31.03.2017

Accepted: 8.05.2018 